\title{
Basics of particle therapy II: relative biological effectiveness
}

\author{
Jinhyun Choi, MD*, Jin Oh Kang, MD \\ Department of Radiation Oncology, Kyung Hee University School of Medicine, Seoul, Korea
}

\begin{abstract}
In the previous review, the physical aspect of heavy particles, with a focus on the carbon beam was introduced. Particle beam therapy has many potential advantages for cancer treatment without increasing severe side effects in normal tissue, these kinds of radiation have different biologic characteristics and have advantages over using conventional photon beam radiation during treatment. The relative biological effectiveness (RBE) is used for many biological, clinical endpoints among different radiation types and is the only convenient way to transfer the clinical experience in radiotherapy with photons to another type of radiation therapy. However, the RBE varies dependent on the energy of the beam, the fractionation, cell types, oxygenation status, and the biological endpoint studied. Thus this review describes the concerns about RBE related to particle beam to increase interests of the Korean radiation oncologists' society.
\end{abstract}

Keywords: Carbon, Proton, Particle beam therapy, Relative biological effectiveness

\section{Introduction}

Particle beam therapy can offer advantages of biological and physical dose distributions over conventional photon beam therapy. There are potential benefits in the use of particle beam therapy for treating anatomically complex and radioresistant malignant tumors because this therapy does not cause severe side effects in normal tissue [1]. Until today, there has been little interest in particle beam therapy in Korea, especially the use of heavy ion radiobiology. Thus we have described the radiobiology issues related to heavy ion beams in order to increase the interests by the Korean radiation oncologists' society.
A characteristic of photons is a random energy distribution through the media in which the resultant ionization density is homogenous over the entire treatment volume. However, the photon beams generate relatively low ionization levels and many photons are required to achieve relevant therapeutic goals. The particle beams, especially heavy ion beams, provide a different characteristic in the spatial distribution of energy. The track structure produces a radial dose distribution around the ion path, which has 2 steps: the emission of electrons by Coulomb interaction and scatter of the emitted electrons [2]. Indirect DNA damage is found greater in low linear energy transfer (LET) radiation but high LET radiation such as carbon ions, creates greater direct hits [3].

Received 28 September 2011, Revised 23 November 2011, Accepted 2 December 2011.

Correspondence: Jin Oh Kang, MD, Department of Radiation Oncology, Kyung Hee University School of Medicine, 26 Kyungheedae-ro, Dongdaemun-gu, Seoul 130-701, Korea. Tel: +82-2-958-8664, Fax: +82-2-962-3002.

*Any correspondences should be directed to Dr. Choi (E-mail: cjh74@daum.net) since corresponding author, Dr. Kang was deceased before publication.

(c) This is an Open Access article distributed under the terms of the Creative Commons Attribution Non-Commercial License (http://creativecommons.org/ licenses/by-nc/3.0/) which permits unrestricted non-commercial use, distribution, and reproduction in any medium, provided the original work is properly cited. www.e-roj.org 
The ionization process is composed of quasifree electrons, Auger electron and convoy electron. Subsequently, these electrons transfer energy by elastic and inelastic collisions. The ionization cross section of the electrons in water exhibits its maximum at approximately $100 \mathrm{eV}$, which relates to a mean free path of a few nanometers [2]. These electrons that are fast enough to leave the track core ( $\delta$ rays) typically undergo a large number of interactions. Due to those frequent scattering processes, the initial preference of electrons in the forward direction diminishes, resulting in a broad angle of distribution. These different energy distributions of heavy ions with a high local dose is on the order of nanometers resulting in a larger probability of correlated nearby DNA damages, such as single or double strand breaks and base damage.

For heavy ions, the dependence of relative biological effectiveness (RBE) on the various physical and biological properties is greater and cannot be disregarded. But this problem was solved by a number of pragmatic steps and assumptions, the clinical RBE is replaced by an LET-dependent RBE for in vitro data and well-defined conditions and then linked to clinical data by an empirical factor [4]. At clinical institutions, proton therapy is based on the use of an RBE value of 1.1 to 1.2 , while for the heavier carbon ions the RBE distribution in the target volume varies between 2 and 5 [5].

\section{Basic understandings about survival curves}

Poisson statistics: In order explain theoretical models, a basic understanding of statistical derivatives is needed. The basic principle to calculate probability is derived in Poisson statistics. The probability of $n$ event $P(n)$ is given by:

$$
\mathbf{P}(\mathbf{n})=\frac{\left(e^{-x}\right)\left(x^{n}\right)}{n !}
$$

where $x$ is the average number of events, $n$ is the specific number of events and e is Euler's number expressed as:

$$
e=\lim _{n \rightarrow \infty}\left(1+\frac{1}{n}\right)^{n} \approx 2.71828
$$

If the probability of being hit is assumed as a kill or inactivation, the probability of not being hit $P(0)$ is based on the following Poisson relationship

$$
\mathrm{P}(0)=\frac{\left(e^{-1}\right)\left(1^{0}\right)}{0 !}=e^{-1}=0.3679 \approx 37 \%
$$

where $x=1$, and $n=0$.

Equations 4 to 11 of this section are based on the article "From
DNA radiation damage to cell death: theoretical approches" by Ballarini [6] published in Journal of Nucleic Acid 2010, which is an open access article permits unrestricted use, distribution, and reproduction in any medium, provided the original work is properly cited. The target model, which was first developed in 1946 and revised in 1955 [7], had limitations that they made no assumption about the induction and repair of the initial DNA damage; this is now known to play a fundamental role in radiation-induced clonogenic death. Therefore, the linearquadratic (LO) model has been adopted as the model of choice to describe survival curves (Fig. 1) [8]. In 1973, Chadwick and Leenhouts [9] developed a molecular model, which is now known as the LO model.

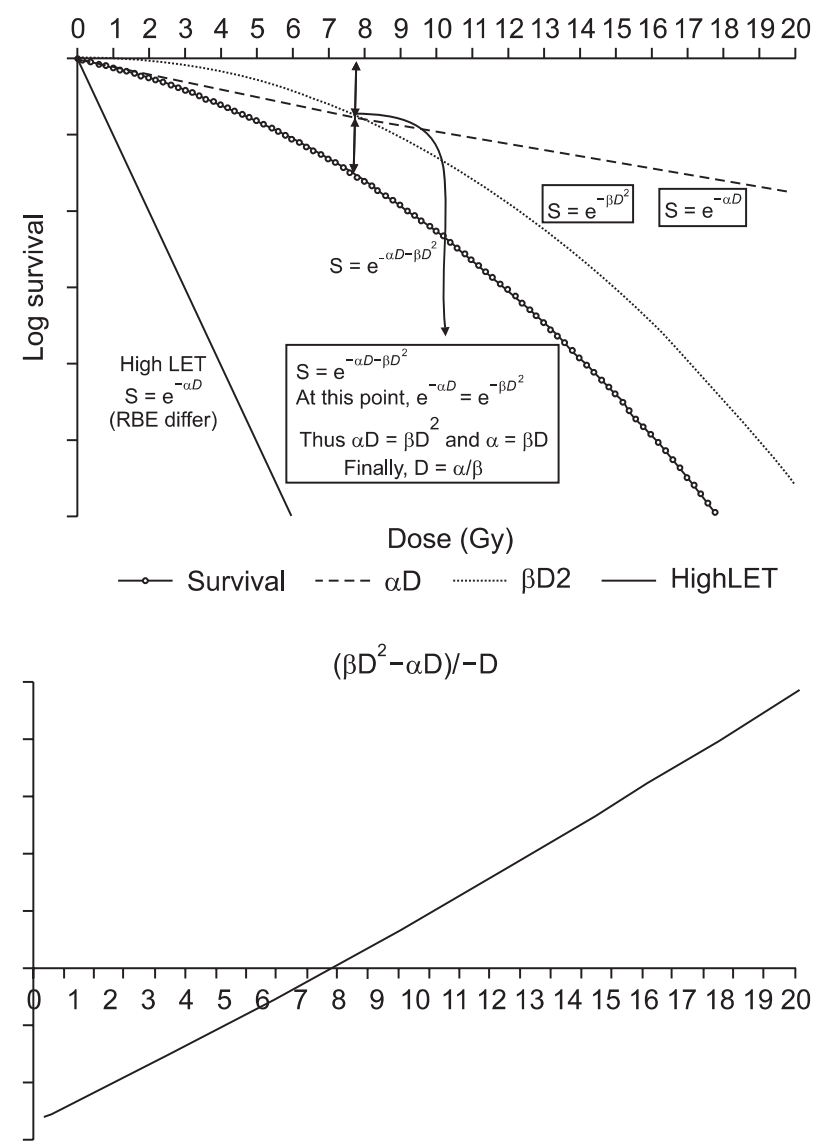

Fig. 1. Linear quadratic survival curve after single dose irradiation. The $\alpha$-component represents non-repairable damage, while the $\beta$-component represents repairable damage. The damage caused by the $\beta$-component is repairable with time, so lowering the fractional dose results in lowering $\beta$-component damage to make $\alpha / \beta$ increase. Theoretically, a very low dose rate (dose/time) radiation resembles $\alpha$ curve. The $\alpha / \beta$ can be plotted when the equation $\left(\beta D^{2}-\alpha D\right) /-D$ intersects the $x$-axis. $R B E$, relative biological effectiveness; LET, linear energy transfer. 
If $N_{0}$ is the number of DNA molecular bond (target), $N$ is the number of the bonds that remain intact after dose $D$, and $K$ is the damage probability of a single bond per unit dose, then

$$
-\frac{d N}{d D}=K N, N=N_{0} e^{-K D}
$$

The number of effective damage (broken bond) is therefore

$$
N_{0}-N=f N_{0}\left(1-e^{-K D}\right)
$$

Where $f$ is the fraction of broken bonds and that are not repaired.

The double helix can undergo a double stands break (DSB) as the result of 2 different mechanisms: (i) both DNA strands are broken by the same radiation track (or event); (ii) each strand is broke independently, but the breaks are close enough in time and space to lead to a DSB. Let $\Delta$ be the fraction of dose acting through mechanism (i), and $(1-\Delta)$ the fraction of dose acting through mechanisms (ii). The number of unrepaired DSBs per cell produced by mechanism (ii) is then

$$
Q_{i i}=E f_{0} q_{1} q_{2}
$$

Where $E$ is the "effective factor", that is the likelihood for a DSB to occur from the 2 single stand breaks (SSBs) associated in time and space, $f_{0}$ is the fraction of unrepaired DSBs, and $q_{1}$ and $q_{2}$ are the number of broken bonds on strand 1 and 2 , respectively. Therefore,

$$
\begin{aligned}
& q_{1}=f_{1} n_{1}\left(1-e^{-k(1-\Delta) D}\right), \\
& q_{2}=f_{2} n_{2}\left(1-e^{-k(1-\Delta) D}\right)
\end{aligned}
$$

Where $n_{1}$ and $n_{2}$ are the number of critical bonds on strand 1 and 2 , respectively, $f_{1}$ and $f_{2}$ are the fractions of unrestored bonds in strand 1 and 2 , and $k$ is the probability of bond rupture per bond and per unit dose.

Adopting a similar notation, the number of unrepaired DSBs induced via mechanism (i) is

$$
Q_{i}=n_{0} f_{0}\left[1-\exp \left(-k_{0} \Delta D\right)\right]
$$

Where $n_{0}$ is the number of DNA sites that can sustain a DSB and $k_{0}$ is the hit probability constant.

The average number of DSBs per cell is therefore $Q_{i}+Q_{i i}$ and the average number of lethal DSBs per cell is

$$
\mathbf{Q}=\mathbf{p}\left(Q_{i}+Q_{i i}\right)
$$

Where $p$ is the assumed proportionality constant between the DSB yield and cell death.

Lumping constant,

$$
\mathbf{Q}=\mathbf{p}\left[\chi\left(1-e^{-k 0 \Delta D}\right)+\varphi\left(1-e^{-k(1-\Delta) D}\right)^{2}\right]
$$

According to Poisson-type cell killing the probability of cell survival $S$ is given by the probability of having 0 lethal lesions, $S=e^{-0}$. Assuming that $k$ and $k_{0}$ are quite small, the result is the familiar linear quadratic relationship as follows:

$$
S=\exp \left(-\alpha D-\beta D^{2}\right)
$$

where $\alpha=\left(\mathrm{f}_{0,} \mathrm{n}_{0}, \mathrm{k}_{0,} \Delta\right)$ and $\beta=\left(\mathrm{f}_{0,}, \mathrm{E}_{1} \mathrm{n}_{1,}, \mathrm{n}_{2,}, \mathrm{f}_{1,}, \mathrm{f}_{2}, \mathrm{k}^{2},(1-\Delta)^{2}\right)$.

By this model, the expression for the survival curves can be drawn as $S=e^{-\left(\alpha D+\beta D^{2}\right)}$, and $S$ is the fraction of cells surviving a dose $\mathrm{D}$. The constants $\alpha$ is used for the component proportional to dose $(\alpha \mathrm{D})$ and the $\beta$ is for the component proportional to the square of the dose $\left(\beta D^{2}\right)$. Survival of the $n$ fractions of dose size $d$ is expressed as $S=e^{-n\left(\alpha d+\beta d^{2}\right)}$. The $\alpha$-component represents non-repairable damage, while the $\beta$-component represents repairable damage. The damage caused by $\beta$-component is repairable with time, so lowering the fractional dose results lowering $\beta$-component damage to increase $\alpha / \beta$. Theoretically, a very low dose rate (dose/time) radiation resembles an $\alpha$ curve.

The final slope of the survival curves of low LET and high LET radiation are similar, while the initial slopes are much different and show that densely ionizing radiation has a high $\alpha$-component; the $\beta$-component remains unchanged [10]. Thus, irradiation of cells with high LET radiation levels produces linear survival curves. Then, for the high LET radiation, the relationship between the surviving fraction $S$ and the dose $D$ is:

$$
\mathbf{S}=e^{-\alpha D}
$$

In the Fig. 1 , the relationship between $D_{0}$ and $\alpha$ is expressed as $D_{0}=1 / \alpha$ because survival after $D_{0}$ is $\mathrm{e}^{-1}$ therefore:

$$
S=e^{-D / D_{0}}
$$

when $D=D_{0}$ 


$$
S=e^{-1}=0.3679 \approx 37 \%
$$

For this reason, the probability of not being hit $\mathrm{P}(0)$ in Poisson statistics can be compared, internally with $D_{0}$ in survival curves, which is often called the mean lethal dose, or the dose that delivers one lethal event per target.

\section{Relative Radiobiological Effectiveness}

The relative biological effectiveness is used to compare biological effectiveness among different types of radiation. In 1990, the International Commission on Radiological Protection (ICRP) advised the use of a weighting factor $\left(W_{R}\right)$ of 1 for low LET radiations by ICRP-60 recommendations [11]. Later on, the ICRP accepted that in vitro experiments on cells show significant differences in radiation quality between Iow LET radiations, but the ICRP continues to recommend a $W_{R}$ of 1 for all low LET radiations [12]. For the high LET radiations, the RBE is used to extrapolate the clinical experiences accumulated from the low LET radiation with the relevant radiation weighting factors. In order to transfer the experiences from photon therapy to particle beam therapy, the RBE is introduced as:

$$
R B E=\frac{D_{\text {photon }}}{D_{\text {particle }}}
$$

$D_{\text {photon }}$ and $D_{\text {particle }}$ are absorbed doses for photon and particle radiation, which lead to the same biological effect, respectively. The biologically effective dose of a photon or particle irradiation is then provided as the product of the absorbed dose multiplied by the RBE. Although it looks simple, the RBE varies with many factors (i.e., LET, energy, particle type, cell type, biological endpoint). These factors cause a serious limitation of the RBE usefulness. Nevertheless, RBE is used because it is the only convenient way to transfer the clinical experience of photon therapy to other types of radiation therapy. The unit of the biological effective dose, which is the Gray-equivalent (GyE) or Cobalt gray equivalent (CGE), if ${ }^{60} \mathrm{Co}$ radiation is used as reference-beam quality. However, the use of these units is not encouraged by the International Commission on Radiation Units \& Measurements - International Atomic Energy Agency (ICRU-IAEA) Task Group, where they recommended the sole use of the term, isoeffective dose, and of the unit, Gy.

The biologic properties of particle beams may differ from those of photons for which the biologic mechanisms have been highly investigated. While the radiobiologic properties of protons are thought to be similar to the photon, the neutrons or heavier ions may produce secondary protons, alpha particles, and heavier nuclear fragments that result in little or no repair of sublethal damage. Therefore, the use of particle therapy has some biological uncertainties that complicate its clinical application. Thus, a fundamental question arises: 'can the biological effect transferred from photons be used as the biological effect of the particle beam', without any reductions in tumor control and/or increase in damage to normal tissue. At the end of 2009, a total of 78,275 patients were treated with particle therapy, and most of whom had been treated with proton therapy $(67,079)$ followed by carbon therapy $(7,151)[13]$. Currently, most proton therapy centers use a generic RBE value of 1.1 in proton treatment planning [14], as recommended by the ICRU [15]. The use of generic RBE means the use of the same RBE in all positions in spread out Bragg peak (SOBP), at all dose levels per fraction and in all tissues. However, application of a single RBE value for protons is complicated because the RBE varies dependent on the energy of the proton [16], the fractionation [17], tissue types [18], oxygenation status [19], and the biological endpoint studied. Though it is widely accepted, a generic RBE cannot be a universal RBE for each tissue or dose/fraction. This is a special concern for young patients who might have secondary malignancy in the future [20]. The RBE can be used for many biological and clinical endpoints derived from DNA strand breaks to tumor cell killing. However, in the scope of particle beam therapy the RBE for cell killing and normal tissue complications are most relevant. Thus the authors discuss the concern about the RBE of particle beams.

\section{Factors Influencing Carbon Beam RBE}

\section{RBE of in vivo tumor model}

As previously described, the RBE depends on dose level and fractionation. RBE is high in lower dose and lower in large dose due to shoulder of photon survival curves [21]. In general, the biological differences between conventional radiation and particle beams are reported to be diminished if the dose is escalated. However, the RBE for clinical situations involves rather large uncertainties, RBEs for each tumor and normal tissues should be calculated for each instance. Thus RBE calculations using biological systems to evaluate RBE relationships at various depths and their dependence on dose per fraction are needed. Unfortunately, the studies addressing 
carbon ion effects in vivo tumor models are relatively small and their endpoints are diverse [22-24]. A recent report for the RBE of carbon ion beams for local tumor control using a syngeneic rat prostate tumor model by Peschke et al. [25] is remarkable. In the report, they set the endpoints of 'no tumor recurrence within 300 days' and volumetric changes after irradiation. With single and split doses of either ${ }^{12} \mathrm{C}$ ions or photons using a 20-mm SOBP the values for $D_{50}$ were used to determine RBE values. A total of 198 rats were irradiated with single and split doses of either photons or carbon ions using a series of increasing dose levels ranging from 30 to $100 \mathrm{~Gy}$ for photon and 9 to $50 \mathrm{~Gy}$ for carbon. The RBEs for the endpoint were $2.30 \pm 0.08$ for single fraction and $2.38 \pm$ 0.16 for two fractions. The experiment showed that there was only a very small fractionation effect for both beam qualities. The $D_{50}$ isoeffect ratio of 1.20 for 2 vs. 1 fraction of photons is not markedly different from that of 1.16 for carbon ions. The reports suggest that the RBE of any reference point should be calculated independently for each clinical application.

\section{RBE and LET}

In the tissue exposed to heavy ions, the RBE is low in the entrance at high energy and increases at the end of the particle range [26]. As the higher ionization density in the track center of particles with a larger LET, the radiation damage became more severe, and thus increasing the RBE. RBE increases with LET up to an ion-dependent maximum value and decreases for higher LET values [27]. It has been reported that the RBE increases as a function of LET up to a maximum value which, for high Z high energy (HZE) charged particles, is found at LET values between 100 and $250 \mathrm{keV} / \mathrm{mm}$ [28]. Koike et al. [24] reported RBEs in animal tumor models using NFSa fibrosarcoma model in mice with different LET. In this study, a clear relationship between RBE and LET was documented. They treated fractionated doses of $290 \mathrm{MeV} / \mathrm{u}$ carbon ions. With LET of 14 and $20 \mathrm{keV} / \mathrm{mm}$, RBEs were 1.4 and independent of the number of fractions, whereas those for 44 and $74 \mathrm{keV} /$ $\mathrm{mm}$, the RBE was increased from 1.8 to 2.3 and from 2.4 to 3.0, respectively, when the number of fractions changed from 1 to 4 . Raising the number of fractions from 4 to 6 was not associated with a further increase in RBE. In another report, Suzuki et al. [29] irradiated sixteen different human cell lines with carbon beam of two different LET values. They calculated RBE values by the $D_{10}$, which is determined as the dose required to reduce the surviving fraction to $10 \%$, relative to X-rays. The calculated RBE ranged from 1.06 to 1.33 for $13-$
keV/microm-beam and from 2.00 to 3.01 for approximate 77 $\mathrm{keV} /$ microm-beam irradiation on each cell line. There was a good correlation in the $D_{10}$ values of each cell line between $X$-rays and carbon-ion beams and the cellular radiosensitivity of different cell lines well correlated among different types of radiation. Notably, two normal cell lines were included in the experiments, suggesting there was no trend for a higher RBE for tumor than normal cell lines. However, the clinical experience with neutron therapy, which is a representative high LET radiation, showed a high RBE for late responding normal tissues (low $\alpha / \beta$ ) evidenced by the increased frequency of late complications. Thus a carbon irradiation would be predicted to be more effective against slowly growing and low $\alpha / \beta$ tumors, vide infra [30].

\section{RBE and cell type}

RBE varies with the nature of cellular characteristics. Weyrather et al. [21] irradiated three hamster cell lines of differing repair capacity with monoenergetic carbon ions. RBE values for cell inactivation at different survival levels were determined and the differences in the RBE-LET patterns were compared with the individual sensitivity. Three hamster cell lines, the wild-type cell lines V79 and Chinese hamster ovary ( $\mathrm{CHO})-\mathrm{K} 1$ and the radiosensitive $\mathrm{CHO}$ mutant $\mathrm{xrs} 5$, were irradiated with carbon ions of LET values (13.7-482.7 keV/ microm). For the repair-proficient cell lines a RBE maximum was found at LET values between 150 and $200 \mathrm{keV} /$ microm. For the repair-deficient cell line the RBE failed to show a maximum and decreased continuously for LET values above $100 \mathrm{keV} /$ microm. The carbon RBE-LET relationship for inactivation is shifted to higher LET values and the RBE correlated with the repair capacity of the cells. Belli et al. [31] irradiated two cancer cell lines and three normal tissue cell lines to compare RBE of monoenergetic carbon-ion beams (incident LET 13$303 \mathrm{keV} / \mu \mathrm{m})$ with three dose-averaged LET values (40, 50, and $75 \mathrm{keV} / \mu \mathrm{m})$. In the report, the data showed the superior effectiveness for cell-killing exhibited by carbon-ion beams compared to lower LET radiation, particularly in tumor cells radioresistant to $X$ - or $\gamma$-rays, hence the advantage of using such beams in radiotherapy. These reports suggest that the variation of cellular nature may result in RBE changes for cellkilling that should be exploited before clinical application. From radiobiological studies, carbon beam treatment may show a high RBE for cell-killing in cells that are repair proficient and radioresistant to $X$ - or $\gamma$-rays [32] but also may imply a greater risk in normal tissue for late effects than $X$ - and $\gamma$-rays 
or protons [33]. Preclinical studies for interested normal or tumor cell lines are therefore fundamental for assessing these radiobiological properties of different cell and tissues.

\section{RBE and particle type}

In clinical setting, the carbon ions are much faster than protons resulting in broader tracks with a reduced ion density in the track center so that at the same LET range, the cell killing effect of carbon ions is smaller than protons. In order to achieve the maximum RBE for the carbon ion, slower ions velocity with a correspondingly smaller tack size and higher LET are required. Likewise, for heavier particles, the RBE maximum is typically sifted to higher LETs. Because the LET sharply increases at the end of the particle's path, forming the Bragg peak, whereas the shape of Bragg peak depends on the type of particles. However, when plotted against dose averaged $L E T, R B E$ distributed so widely that no relation between LET and RBE could be found except a trend showing large RBE at around 80-200 keV/mm [34]. George et al. [35] reported chromosome aberrations of human lymphocytes after in vitro exposure to ${ }^{1} \mathrm{H},{ }^{3} \mathrm{He},{ }^{12} \mathrm{C},{ }^{40} \mathrm{Ar}_{1}{ }^{28} \mathrm{Si}_{1}{ }^{56} \mathrm{Fe}$, or ${ }^{197} \mathrm{Au}$ ion beams, with LET ranging from approximately $0.4-1,393 \mathrm{keV} /$ microm in the dose range of $0.075-3 \mathrm{~Gy}$. In the report, the estimates of RBEmax values for mitotic spreads, which ranged from near 0.7 to 11.1 for total exchanges, increased with LET, reaching a maximum at about $150 \mathrm{keV} /$ microm, and decreased with further increase in LET. Han et al. [36] investigated primary Syrian hamster embryo (SHE) cell transformation by silicon ions and carbon ions. In the report, the RBE for carbon ions ranged from 2.2 to 6.9 and for silicon ions ranged from 2.0 to 6.0. Czub et al. [37] compared RBE for cell inactivation and LET in the Bragg peak region of ${ }^{12} \mathrm{C}$ and ${ }^{20} \mathrm{Ne}$ ions. $\mathrm{CHO}-\mathrm{K} 1$ cells were exposed to high LET ${ }^{12} \mathrm{C}$ ions (33.2 MeV, 20.3 MeV, and 9.1 $\mathrm{MeV}$ at cell entrance) and ${ }^{20} \mathrm{Ne}$ ions (56.2 MeV, 34.7 MeV, and $15 \mathrm{MeV}$ at cell entrance) and to low LET x-rays. RBE has been measured for LET values close to the Bragg peak maximum, i.e., 440-830 keV/microm for ${ }^{12} \mathrm{C}$ and for 1,020-1,600 keV/microm for ${ }^{20} \mathrm{Ne}$ ions. The RBE decreases with increasing LET in the range between 440 and 1,600 keV/microm for the two types of radiations forming a single line when plotted together, pointing towards LET as the single determinant of RBE. These reports suggest that the RBE varies with particle type end biological endpoints, however, when they plotted against dose averaged LET, the RBE of heavy particles varies so widely that no clear relationship can be made between particle types and RBE.

\section{RBE of Carbon Beam}

\section{Single fractionation}

The RBE of high LET radiation is defined as the ratio of the tested high LET radiation dose to that required from low LET radiation to achieve that effect $[10,38]$. The following equations are modified from the equations of Dale et al. [38].

$$
R B E=\frac{d_{L}}{d_{H}}
$$

Where $d_{L}$ is dose of low LET and $d_{H}$ is dose of high LET radiation.

The survival of low LET radiation and high LET radiation are expressed as:

$$
\begin{aligned}
& S_{L}=\exp \left(-\alpha_{L} d_{L}-\beta_{L} d_{L}^{2}\right) \\
& S_{H}=\exp \left(-\alpha_{H} d_{H}-\beta_{H} d_{H}^{2}\right)
\end{aligned}
$$

Thus the logarithms of the survival become

$$
\begin{aligned}
& \ln S_{L}=-d_{L}\left(\alpha_{L}+\beta_{L} d_{L}\right), \\
& \ln S_{H}=-d_{H}\left(\alpha_{H}+\beta_{H} d_{H}\right)
\end{aligned}
$$

Then,

$$
d_{L}=-\frac{\ln S_{L}}{\left(\alpha_{L}+\beta_{L} d_{L}\right)}, d_{H}=-\frac{\ln S_{H}}{\left(\alpha_{H}+\beta_{H} d_{H}\right)}
$$

Then the RBE is expressed as:

$$
R B E=\frac{\frac{\ln S_{L}}{\left(\alpha_{L}+\beta_{L} d_{L}\right)}}{\frac{\ln S_{H}}{\left(\alpha_{H}+\beta_{H} d_{H}\right)}}
$$

If the fractional dose get closer to $0(D \rightarrow 0)$, In $S_{L}$ and $\ln S_{H}$ become $1\left(10^{\circ}\right)$ and the $\beta$ contribution becomes minimal ( $D \rightarrow 0$, $\beta \rightarrow 0$ ). It can be expressed as

$$
R B E_{\beta \rightarrow 0}=\frac{\alpha_{H}}{\alpha_{L}}
$$

If the fractional dose becomes large enough, the In $S_{L}$ becomes closer to $\ln S_{H_{1}}$ and $\alpha$ contribution becomes minimal $(\mathrm{D} \rightarrow \infty, \alpha \rightarrow 0)$. Thus it can be expressed as:

$$
\beta_{L} d_{L}^{2}=\beta_{H} d_{H}^{2}
$$




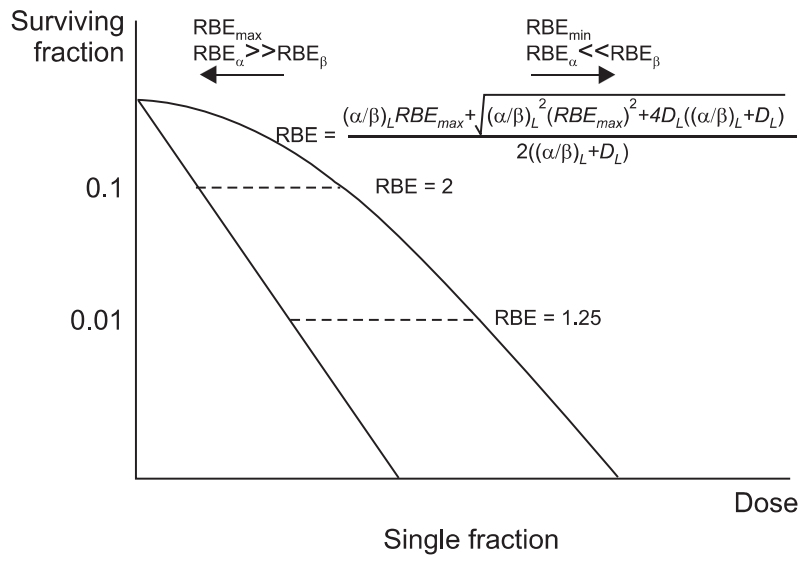

Fig. 2. Relative biological effectiveness (RBE) after single dose irradiation. If the fractional dose get closer to $0(D \rightarrow 0), \ln S_{L}$ and In $S_{H}$ become $1\left(10^{\circ}\right)$ and the $\beta$ contribution becomes minimal $(D \rightarrow 0, \beta \rightarrow 0)$. If the fractional dose becomes large enough, the In $S_{L}$ becomes closer to In $S_{H_{1}}$ and $\alpha$ contribution becomes minimal $(\mathrm{D} \rightarrow \infty, \alpha \rightarrow 0)$. According to linear quadratic model, RBE is maximal when dose $\rightarrow 0$. If the fractional dose increases, the RBE converges to a minimal value.

$$
\frac{d_{L}^{2}}{d_{H}^{2}}=\frac{\beta_{H}}{\beta_{L}}
$$

$$
R B E_{\alpha \rightarrow 0}=\sqrt{\frac{\beta_{H}}{\beta_{L}}}
$$

According to LO model, RBE is maximal when dose $\rightarrow 0$. At this point the $\alpha$-component occupies the most effective component of the biologic effect. If the fractional dose increases, the RBE converges to a minimal value. $R B E_{\beta \rightarrow 0}$ is $R B E_{\max }$ and $R B E_{\alpha \rightarrow 0}$ is $R B E_{\min }$ (Fig. 2). This suggests that the difference between the RBEs is reduced with larger doses per fraction. However, larger fractional dose is related to an increase in complications.

$$
\begin{aligned}
& R B E_{d \rightarrow 0}=R B E_{\max }=\frac{\alpha_{H}}{\alpha_{L}} \\
& \alpha_{H}=\alpha_{L} R B E_{\max }
\end{aligned}
$$

With the concept that the RBE means the same biological effect in both low LET and high LET radiation, $\ln S_{L}=\ln S_{H}$ Thus,

$$
\begin{aligned}
& \alpha_{L} D_{L}+\beta_{L} D_{L}^{2}=\alpha_{L} R B E_{\max } D_{H}+\beta_{H} D_{H}^{2} \\
& \alpha_{L} R B E_{\max } D_{H}=\left(\alpha_{L} D_{L}+\beta_{L} D_{L}^{2}\right)-\beta_{H} D_{H}^{2}
\end{aligned}
$$

$$
R B E_{\text {max }}=\frac{\left(\alpha_{L} D_{L}+\beta_{L} D_{L}^{2}\right)-\beta_{H} D_{H}^{2}}{\alpha_{L} D_{H}}
$$

When dose $\rightarrow 0$, the $\beta$ contribution goes to negligible level and we can assume $\beta_{H}=\beta_{L}$. The equations divided with $\beta_{L^{\prime}}$

$$
\begin{aligned}
& \boldsymbol{R B} E_{\text {max }}=\frac{\left((\alpha / \beta){ }_{L} D_{L}+D_{L}^{2}\right)-D_{H}^{2}}{(\alpha / \beta){ }_{L} D_{H}} \\
& R B E_{\text {max }}=\frac{D_{L}\left((\alpha / \beta){ }_{L}+D_{L}\right)-D_{H}^{2}}{(\alpha / \beta)_{L} D_{H}}
\end{aligned}
$$

From equation 27,

$$
\begin{aligned}
& \left(\alpha_{L} D_{L}\right) / D_{H}+\left(\beta_{L} D_{L}^{2}\right) / D_{H}= \\
& \left(\alpha_{L} R B E_{\max } D_{H}\right) / D_{H}+\left(\beta_{H} D_{H}^{2}\right) / D_{H} \\
& \alpha_{L} R B E+\beta_{L} D_{L} R B E=\alpha_{L} R B E_{\max }+\beta_{H} D_{H} \\
& \alpha_{L} R B E+\beta_{L} D_{L} R B E=\alpha_{L} R B E_{\max }+\beta_{H} \frac{D_{L}}{R B E} \\
& \alpha_{L} R B E^{2}+\beta_{L} D_{L} R B E^{2}=\alpha_{L} R B E_{\max } R B E+\beta_{H} D_{L} \\
& \left(\alpha_{L}+\beta_{L} D_{L}\right) R B E^{2}-\alpha_{L} R B E_{\max } R B E-\beta_{H} D_{L}=0 \\
& R B E=\frac{\alpha_{L} R B E_{\max }+\sqrt{\left(\alpha_{L} R B E_{\max }\right)^{2}+4\left(\alpha_{L}+\beta_{L} D_{L}\right)\left(\beta_{H} D_{L}\right)}}{2\left(\alpha_{L}+\beta_{L} D_{L}\right)}
\end{aligned}
$$

When dose $\rightarrow 0$, the $\beta$ contribution goes to negligible level and we can assume $\beta_{H}=\beta_{L}$.

$$
R B E=\frac{\alpha_{L} R B E_{\max }+\sqrt{\left(\alpha_{L} R B E_{\max }\right)^{2}+4\left(\alpha_{L}+\beta_{L} D_{L}\right)\left(\beta_{L} D_{L}\right)}}{2\left(\alpha_{L}+\beta_{L} D_{L}\right)}
$$

Divide with $\beta_{L}$.

$$
R B E=\frac{(\alpha / \beta)_{L} R B E_{\max }+\sqrt{(\alpha / \beta)_{L}^{2}\left(R B E_{\max }\right)^{2}+4 D_{L}\left((\alpha / \beta)_{L}+D_{L}\right)}}{2\left((\alpha / \beta)_{L}+D_{L}\right)}
$$

\section{Multiple fractionation}

In 2008, Dasu and Toma-Dasu [39] reported a clinical relevant RBE model warning about fractionated therapy with high LET radiation. The reliability on the transfer of tolerance and curative levels from low LET radiation to high LET radiation by using RBE was questioned. They introduced the dose modifying factor (DMF) concept to simplify the formula (Fig. 3). The effects of high LET radiation can be described using similar 


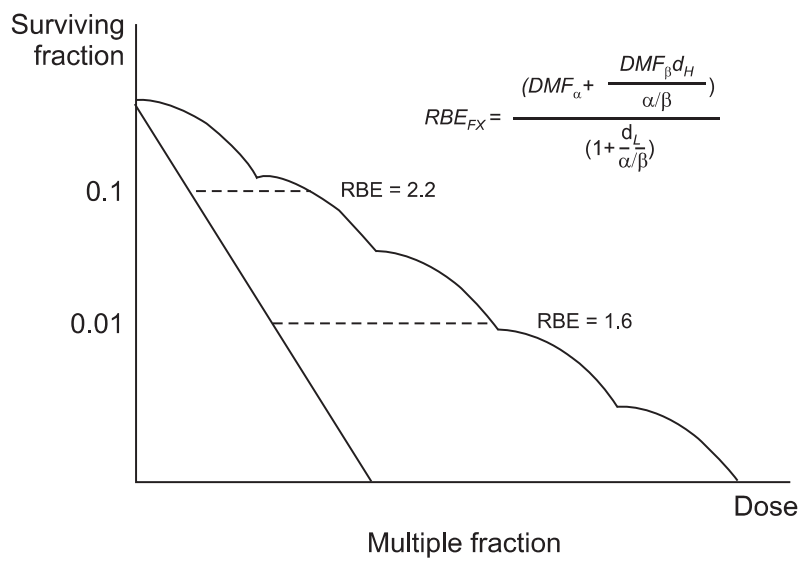

Fig. 3. Relative biological effectiveness (RBE) after multiple fractionation. The effects of high linear energy transfer (LET) radiation can be described using similar equations, either by using different $\alpha$ and $\beta$ parameters or by applying dose modifying factors (DMF) to the parameters describing the response to low LET radiation. The DMF is drawn by experimental results.

equations, either by using different $\alpha$ and $\beta$ parameters or by applying DMF to the parameters describing the response to Iow LET radiation. DMF is drawn by experimental data.

A brief citing of their formula is that RBE means the ratio of the low LET dose to high LET dose with 'same biologic effect' so that it can be expressed as

$$
\begin{aligned}
& \ln S_{L}=\ln S_{H} \\
& \alpha D_{L}+\beta D_{L}^{2}=\alpha D M F_{\alpha} D_{H}+\beta D M F_{\beta} D_{H}^{2} \\
& \beta D_{L}^{2}+\alpha D_{L}-\left(\alpha D M F_{\alpha} D_{H}+\beta D M F_{\beta} D_{H}^{2}\right)=0 \\
& \text { let }\left(\alpha D M F_{\alpha} D_{H}+\beta D M F_{\beta} D_{H}^{2}\right)=\mathrm{C} \\
& \beta D_{L}^{2}+\alpha D_{L}-\mathrm{C}=\mathbf{0}
\end{aligned}
$$

by quadratic equation,

$$
D_{L}=\frac{-\alpha \pm \sqrt{\alpha^{2}+4 \beta C}}{2 \beta}
$$

$$
\begin{aligned}
& R B E_{F X}=\frac{D_{L}}{D_{H}}=\frac{-\alpha \pm \sqrt{\alpha^{2}+4 \beta C}}{2 \beta \cdot D_{H}} \\
& =\frac{-\alpha \pm \sqrt{\alpha^{2}+4 \beta\left(\alpha D M F_{\alpha} D_{H}+\beta D M F_{\beta} D_{H}^{2}\right)}}{2 \beta \cdot D_{H}}
\end{aligned}
$$

Thus RBE can be drawn by the factors of high LET radiation.

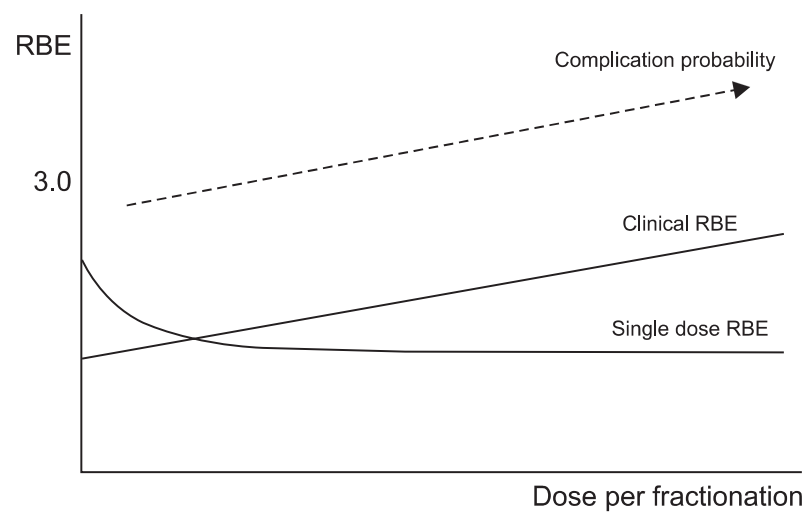

Fig. 4. The relative biological effectiveness (RBE) increases with fractionated radiation in contrast to the single fraction radiation (modified from Dasu and Toma-Dasu [39]). The complication probability of spread out Bragg peak may increase also (modified from Karger et al. [40]).

For the RBE of fractionated radiation, a similar approach can be used.

$$
\begin{aligned}
& \mathbf{n}\left(\alpha d_{L}+\beta d_{L}^{2}\right)=\mathbf{n}\left(\alpha D M F_{\alpha} d_{H}+\beta D M F_{\beta} d_{H}^{2}\right) \\
& d_{L}\left(\alpha+\beta d_{L}\right)=d_{H}\left(\alpha D M F_{\alpha}+\beta D M F_{\beta} d_{H}\right) \\
& R B E_{F X}=\frac{D_{L}}{D_{H}}=\frac{\left(\alpha D M F_{\alpha}+\beta D M F_{\beta} d_{H}\right)}{\left(\alpha+\beta d_{L}\right)}=\frac{\left(D M F_{\alpha}+\frac{D M F_{\beta} d_{H}}{\alpha / \beta}\right)}{\left(1+\frac{d_{L}}{\alpha / \beta}\right)}
\end{aligned}
$$

For details on the derivation of the above formula, further reading is recommended [39].

With this equation, the authors compared the RBE of single fractionation and multiple fractionations. The authors showed that the clinically relevant RBE increased for greater doses per fraction when multiple fractions were applied, in contrast to the predictions from single-dose experiments (Fig. 4). Furthermore, the RBE of late-reacting tissues appeared to modify more quickly than that for early-reacting tissues. The authors concluded that the modification of the RBE with dose per fraction must be carefully taken into consideration when devising fractionated treatments with high LET radiation. They also emphasized that neglecting to do so may result in an avalanche of complications that could obscure the potential advantages of the therapeutic use of this radiation type.

The RBE in SOBP may increase further. Karger et al. [40] compared the tolerance of the rat spinal cord to photon and carbon ion irradiations in the plateau region and in a $1-\mathrm{cm}$ SOBP. Rats were irradiated with 6 or 18 fractions of photons 
or ${ }^{12} \mathrm{C}$-ions, respectively and followed up for 300 days for the onset of paresis grade II and dose-response curves were calculated. The corresponding RBE-values were $1.33 \pm 0.02$, $1.42 \pm 0.02$ ( 6 and 18 fractions, ${ }^{12} \mathrm{C}$-plateau) and $2.97 \pm 0.05$, $5.04 \pm 0.08$ ( 6 and 18 fractions, ${ }^{12} \mathrm{C}$-peak), respectively. The experiment showed that carbon ion irradiations of the spinal cord are significantly more effective in the peak than in the plateau region. In the Bragg-peak, it may underestimates the RBE by $25 \%$.

\section{RBE in Practice: HIMAC vs. GSI}

RBE itself is a simple concept expressed as $D_{\text {photon }} / D_{\text {carbon}}$. But in clinical practice, very complicated extrapolations of concerning factors are required for therapeutic planning because it depends on many factors such as dose [21], LET [27], particle type, and cell types [29]. The RBE differences between conventional radiation and particle beams decreased if the dose is escalated because RBE is high in lower dose and low in large dose due to shoulder of photon survival curves [21]. The RBE varies with LET. RBE increases with LET up to an iondependent maximum value and decreases at higher LET values [27]. Due to the higher ionization density in the track center, the LET in increased resulting more severe radiation damage, thus the RBE is increased However, as the LET increased further, the number of ions required for the same dose become relatively lower to make the hitting probability decreased (low efficacy). Also, the RBE varies according to the characteristics of tissue or cell type. Suzuki et al. [41] determined the survival curves for more than a dozen lineages of normal and tumor cells. In the report, the RBE values calculated by the $D_{10}$, ranged from 1.06 to 1.33 for low LET radiation, and from 2.00 to 3.01 for high LET radiation. Another characteristic of radiation sensitivity $\alpha / \beta$ ratio showed correlation with RBE, because RBE depends on the $\alpha / \beta$ ratio after conventional photon irradiation so that RBE increases with decreasing $\alpha / \beta$ ratio [42]. Therefore, the RBE of carbon ions may relate to the $\alpha / \beta$ ratio determined by photon. Considering these factors, an effort for clarification and application of clinical RBE were made in the carbon therapy facilities. However, their concepts on carbon RBE were much different.

The heavy-ion medical accelerator complex (HIMAC), which first started heavy ion therapy, used experimental data combined with clinical neutron experience data. At first, they calculated the depth dose distribution and LET distribution of a monoenergetic carbon beam. In vitro survival experiments using mono-energetic carbon ions were performed. Then a dose weighted average based algorithm was set. Then relative shape of depth dose distribution curve was made [29]. It was found that the neutron RBE at several survival levels coincided with the RBE of the carbon beam at a dose averaged LET around $65 \mathrm{keV} / \mu \mathrm{m}$. Thus they assumed that the neutron beam is nearly equivalent to a $65 \mathrm{keV} / \mu \mathrm{m}$ carbon beam. As a next step, a clinical neutron experience was combined to determine absolute RBE [4]. As a result, a clinical carbon RBE at the center of the SOBP was determined as 2.38 [4]. Finally, it was noticed that the RBE for the inactivation of human salivary gland cells of a carbon SOBP at the $80 \mathrm{keV} / \mu \mathrm{m}$ position coincides with the RBE of a neutron beam. But this cellular RBE $=2$ was much smaller than the clinical neutron $\mathrm{RBE}=3$. Consequently, for the clinical treatment planning of carbon beams the RBE at the $80 \mathrm{keV} / \mu \mathrm{m}$ position was fixed to the neutron value RBE $=3$, independently from dose and fractionation schedule. Currently, the National Institute of Radiological Sciences (NIRS) is developing a model based on spot scanning system, which is totally different from the passive scanning system that is used first carbon therapy in HIMAC.

The Gesellschaft fur Schwerionenforschung (GSI) developed their own biophysical model called local effect model (LEM). The basic assumption of GSI model is that biological damage is determined by local dose and there is no qualitative difference since the damage is generated by ejected delta electrons. The only difference is that local distribution of dose. The principle of local effect model is:

Local biological effect $\mathrm{S}$ is

$$
\mathbf{S}=e^{-\bar{N}_{\text {lethal }}}
$$

Low LET dose response is

$$
\bar{N}_{\text {lethal }}(D)=-\ln S_{x}(D)=\alpha D+\beta D^{2}
$$

The event density is

$$
v(\mathrm{D})=\bar{N}_{\text {lethal }}(\mathrm{D}) / V_{\text {Nucleus }}
$$

So,

$$
\bar{N}_{\text {lethal }}=\int \frac{-\ln S_{x}(d(x, y, z))}{V_{N u c l e u s}} d V_{N u c l e u s}
$$

Currently the LEM III is reported where the description of track structure is modified by taking into account a velocity- 
dependent extension of the inner part of the track to improve the accuracy [42]. They reported that the original version of the LEM (LEM I) underestimates the therapeutic ratio of carbon ions (i.e., the ratio of RBE in the Bragg peak region as compared with the $\mathrm{RBE}$ in the entrance channel). Although significantly reduced, the cluster extension of the LEM (LEM II) still shows the same tendency. Implementation of the modified track structure (LEM III) almost completely compensates these systematic deviations, and predictions of RBE by LEM III for high and low energetic carbon ions show good agreement for a wide panel of different cell lines, as well as for the tolerance of the rat spinal cord. As a consequence, the expected RBE in the normal tissue surrounding the tumor becomes significantly lower than estimated with the LEM in its original version (LEM I). They concluded that LEM III model will be particularly useful for further optimization of carbon ion therapy in general.

Recently, the RBE of HIMAC model and GSI model were directly compared [43]. Even with the different concepts of two models and different methods for beam delivery of two institutes, the RBE difference was not big in the report. They compared biological effectiveness of $290 \mathrm{MeV} / \mathrm{amu}$ carbonion beams in Chiba, Japan and in Darmstadt, Germany. Murine small intestine and human salivary gland tumor (HSG) cells exponentially growing in vitro were irradiated with 6-cm width of SOBPs adjusted. Cobalt-60 gamma rays were used as the reference radiation. Isoeffective doses at given survivals were used for RBE calculations and inter-institutional comparisons. As a result, isoeffective $D_{10}$ doses of HSG cells ranged from 2.37 $\pm 0.14 \mathrm{~Gy}$ to $3.47 \pm 0.19 \mathrm{~Gy}$ for Chiba and from $2.31 \pm 0.11 \mathrm{~Gy}$ to $3.66 \pm 0.17$ Gy for Darmstadt. Isoeffective $D_{10}$ doses of gut crypts after single doses ranged from $8.25 \pm 0.17$ Gy to 10.32 \pm 0.14 Gy for Chiba and from $8.27 \pm 0.10$ Gy to $10.27 \pm 0.27$ Gy for Darmstadt, whereas isoeffective $D_{30}$ doses after three fractionated doses were $9.89 \pm 0.17$ Gy through $13.70 \pm 0.54$ Gy and $10.14 \pm 0.20$ Gy through $13.30 \pm 0.41$ Gy for Chiba and Darmstadt, respectively. Overall difference of RBE between the two facilities was $0-5 \%$ or $3-7 \%$ for gut crypt survival or HSG cell killing, respectively. They concluded that the carbon-ion beams at the NIRS in Chiba, Japan and the GSI are biologically identical after single and daily fractionated irradiation.

\section{Proton RBE}

\section{Beam modulation system}

Proton serves advantages with their physical dose distribution which may provide reduced side effects and increased tumor control probability through dose escalation. In clinical practice, the variation of RBE within the SOBP is regarded as negligible in order to have 1.1 as the RBE value. However, as the SOBP is formed by multiple superimposed plateaus and peaks of varying intensity and range, the LETs in the proximal and distal portion of the SOBP must be different. In the proximal area, the proton is of a higher energy and lower LET, while in the distal area the energy lowers to make the LET higher. Meanwhile, the proton beam therapy delivers relatively higher fractional dose for target volumes, and in many cases critical normal structures are close to the volume. As the dose prescribed for a patient is directly related to the selected clinical RBE, an accuracy of $5 \%$ is also required for the determination of the RBE [44]. With exceptions, the available experimental data indicate that the RBE of SOBP protons increases with decreasing dose or dose per fraction and the increasing depth in the SOBP, with the magnitude of both effects likely being dependent on the $\alpha / \beta$ ratios of the target cells or tissues [45]. The variations in SOBP are greater in proton therapy with passive beam modulation system. In the passive modulation system, the particle beam is adapted in 3 dimensions to the target volume only by passive non-variable field shaping elements. The beam shaping devices, such as the brass aperture and range compensator, are the sources of undesired secondary radiation. Meanwhile, the active beammodulation system, a fine pencil-like beam is used to fill the voxels, which compose the target volume thus the path of the proton beam is not disturbed [46]. This active scanning technique is expected to produce less out-of-field dose when treating the same target volume [20]. In the aspect of RBE in clinical practice, the passive modulation system serves simple way to direct the $R B E$, because the RBE depth profile remains constant over the treatment field [4]. Thus, the RBE profile can directly be considered in the design of the modulator wheel in the passive modulation system. The active-modulation system is more sophisticated and theoretically, the RBE should be calculated separately for each voxel within the treatment field [47].

\section{Proton RBE in Korea}

In Korea, only one proton therapy facility is currently opera- 
ting at the National Cancer Center. The radiobiological characteristic of the proton beam is reported in 2008 [48]. RBE has been calculated in HSG cells, using clonogenic survival and the MTT assay (cell viability test) at a fixed $190 \mathrm{MeV}$ proton beam with $6 \mathrm{~cm}$ SOBP. The reported RBE was $1.024 \pm 0.007$ by clonogenic assay and $1.049 \pm 0.028$ by MTT assay. In the report, further analysis of the biological response of proton exposure revealed no difference compared to conventional $X$-ray treatment on Western blot, and fluorescent activated cell sorter (FACS) analysis. Although the reported RBE of the center was slightly lower than generic RBE 1.1, they still use generic RBE 1.1 as the representative RBE without correction. In 2011, in vivo RBE was reported from the same institution [49]. In the report they changed energy to $210 \mathrm{MeV}$ with same 6-cm SOBP. The RBE was estimated from the survival of jejunal crypt in $\mathrm{C} 3 \mathrm{H} / \mathrm{He}$ mice. The doses that reduced the number of regenerated crypt per jejunal circumference to $20\left(D_{20}\right)$ in $\mathrm{C} 3 \mathrm{H} / \mathrm{He}$ mice were $14.8 \mathrm{~Gy}$ for $\mathrm{X}$-rays and $13.5 \mathrm{~Gy}$ for protons so that the RBE in the middle of the SOBP was 1.10 in this case.

\section{Outlook}

The reason we use RBEs in practice is to bring our inherited clinical experiences of photon beam era into particle beam treatment. However, the RBEs of particle beams, especially for heavy ions, show much dynamic changes according to various factors. The authors hope this contribution may provoke some biological interests for the Korean radiation oncologists' society on the particle therapies.

\section{Conflict of Interest}

No potential conflict of interest relevant to this article was reported.

\section{Acknowledgments}

This work was supported by Development of Heavy Ion Medical Accelerator Program of Ministry of Education, Science \& Technology (MEST) (grant code: 2011k000567).

\section{References}

1. Fokas $E$, Kraft $G, A n H$, Engenhart-Cabillic R. Ion beam radio- biology and cancer: time to update ourselves. Biochim Biophys Acta 2009;1796:216-29.

2. Schardt D, Elsasser T, Schulz-Ertner D. Heavy-ion tumor therapy: physical and radiobiological benefits. Rev Mod Phys 2010;82:383-425.

3. Ito $A$, Nakano $H$, Kusano $Y$, et al. Contribution of indirect action to radiation-induced mammalian cell inactivation: dependence on photon energy and heavy-ion LET. Radiat Res 2006;165:703-12.

4. Kanai T, Endo M, Minohara S, et al. Biophysical characteristics of HIMAC clinical irradiation system for heavy-ion radiation therapy. Int J Radiat Oncol Biol Phys 1999;44:201-10.

5. Skarsgard LD. Radiobiology with heavy charged particles: a historical review. Phys Med 1998;14 Suppl 1:1-19.

6. Ballarini F. From DNA radiation damage to cell death: theoretical approaches. J Nucleic Acids 2010;2010:350608.

7. Lea DE. Actions of radiations on living cells. 2nd ed. London: Cambridge University Press; 1955.

8. Hall EJ, Giaccia AJ. Radiobiology for the radiologist. 6th ed. Philadelphia, PA: Lippincott Williams \& Wilkins; 2006.

9. Chadwick KH, Leenhouts HP. A molecular theory of cell survival. Phys Med Biol 1973;18:78-87.

10. Dale RG, Jones B. The assessment of RBE effects using the concept of biologically effective dose. Int J Radiat Oncol Biol Phys 1999;43:639-45.

11. ICRP. 1990 recommendations of the International Commission on Radiological Protection. Ann ICRP 1991;21(1-3):1-201.

12. ICRP. The 2007 recommendations of the International Commission on Radiological Protection: ICRP publication 103. Ann ICRP 2007;37(2-4):1-332.

13. Particle Therapy Co-operative Group (PTCOG). Patient statistics per end of 2010 [Internet]. PTCOG; 2011 [cited 2011 Mar 20]. Available from: http://ptcog.web.psi.ch/patient_statistics.html.

14. Paganetti $H$, Niemierko $A$, Ancukiewicz $M$, et al. Relative biological effectiveness (RBE) values for proton beam therapy. Int J Radiat Oncol Biol Phys 2002;53:407-21.

15. International Commission on Radiation Units and Measurements (ICRU). Prescribing, recording, and reporting protonbeam therapy. J ICRU 2007;7:1-210.

16. Wambersie A. RBE, reference RBE and clinical RBE: applications of these concepts in hadron therapy. Strahlenther Onkol 1999;175 Suppl 2:39-43.

17. Gueulette J, Slabbert JP, Bohm L, et al. Proton RBE for early intestinal tolerance in mice after fractionated irradiation. Radiother Oncol 2001;61:177-84.

18. Paganetti H, Gerweck LE, Goitein M. The general relation between tissue response to $\mathrm{x}$-radiation (alpha/beta-values) and the relative biological effectiveness (RBE) of protons: prediction by the Katz track-structure model. Int J Radiat Biol 
2000;76:985-98.

19. Lee KB, Lee JS, Park JW, Huh TL, Lee YM. Low energy proton beam induces tumor cell apoptosis through reactive oxygen species and activation of caspases. Exp Mol Med 2008;40:11829.

20. Hall EJ. Intensity-modulated radiation therapy, protons, and the risk of second cancers. Int J Radiat Oncol Biol Phys 2006;65:1-7.

21. Weyrather WK, Ritter S, Scholz M, Kraft G. RBE for carbon track-segment irradiation in cell lines of differing repair capacity. Int J Radiat Biol 1999;75:1357-64.

22. Takahashi A, Yano T, Matsumoto $H$, et al. Effects of accelerated carbon-ions on growth inhibition of transplantable human esophageal cancer in nude mice. Cancer Lett 1998;122:181-6.

23. Kitabayashi H, Shimada H, Yamada S, et al. Synergistic growth suppression induced in esophageal squamous cell carcinoma cells by combined treatment with docetaxel and heavy carbon-ion beam irradiation. Oncol Rep 2006;15:913-8.

24. Koike $S$, Ando K, Oohira C, et al. Relative biological effectiveness of $290 \mathrm{MeV} / \mathrm{u}$ carbon ions for the growth delay of a radioresistant murine fibrosarcoma. J Radiat Res 2002;43:24755.

25. Peschke $P$, Karger CP, Scholz M, Debus J, Huber PE. Relative biological effectiveness of carbon ions for local tumor control of a radioresistant prostate carcinoma in the rat. Int $\mathrm{J}$ Radiat Oncol Biol Phys 2011;79:239-46.

26. Kramer M, Weyrather WK, Scholz M. The increased biological effectiveness of heavy charged particles: from radiobiology to treatment planning. Technol Cancer Res Treat 2003;2:427-36.

27. Furusawa $Y$, Fukutsu $K_{1}$, Aoki $M$, et al. Inactivation of aerobic and hypoxic cells from three different cell lines by accelerated (3)He-, (12)C- and (20)Ne-ion beams. Radiat Res 2000;154: 485-96.

28. Kraft G. Tumor therapy with heavy charged particles. Prog Part Nucl Phys 2000;45:S473-544.

29. Suzuki M, Kase $Y$, Yamaguchi $H$, Kanai T, Ando K. Relative biological effectiveness for cell-killing effect on various human cell lines irradiated with heavy-ion medical accelerator in Chiba (HIMAC) carbon-ion beams. Int J Radiat Oncol Biol Phys 2000;48:241-50.

30. Suit $H$, DeLaney $T$, Goldberg $S$, et al. Proton vs carbon ion beams in the definitive radiation treatment of cancer patients. Radiother Oncol 2010;95:3-22.

31. Belli $M$, Bettega $D$, Calzolari $P$, et al. Effectiveness of monoenergetic and spread-out bragg peak carbon-ions for inactivation of various normal and tumour human cell lines. J Radiat Res 2008;49:597-607.

32. Schulz-Ertner D, Jakel O, Schlegel W. Radiation therapy with charged particles. Semin Radiat Oncol 2006;16:249-59.
33. Blakely EA, Chang PY. Late effects from hadron therapy. Radiother Oncol 2004;73 Suppl 2:S134-40.

34. Ando K, Kase Y. Biological characteristics of carbon-ion therapy. Int J Radiat Biol 2009;85:715-28.

35. George K, Durante M, Willingham V, et al. Biological effectiveness of accelerated particles for the induction of chromosome damage measured in metaphase and interphase human lymphocytes. Radiat Res 2003;160:425-35.

36. Han ZB, Suzuki H, Suzuki F, et al. Relative biological effectiveness of accelerated heavy ions for induction of morphological transformation in Syrian hamster embryo cells. J Radiat Res 1998;39:193-201.

37. Czub J, Banas D, Blaszczyk A, et al. Biological effectiveness of (12) C and (20)Ne ions with very high LET. Int J Radiat Biol 2008;84:821-9.

38. Dale RG, Jones B, Carabe-Fernandez A. Why more needs to be known about RBE effects in modern radiotherapy. Appl Radiat Isot 2009;67:387-92.

39. Dasu A, Toma-Dasu I. What is the clinically relevant relative biologic effectiveness? a warning for fractionated treatments with high linear energy transfer radiation. Int J Radiat Oncol Biol Phys 2008;70:867-74.

40. Karger CP, Peschke P, Sanchez-Brandelik R, Scholz M, Debus J. Radiation tolerance of the rat spinal cord after 6 and 18 fractions of photons and carbon ions: experimental results and clinical implications. Int J Radiat Oncol Biol Phys 2006;66:1488-97.

41. Suzuki M, Kase Y, Kanai T, Ando K. Correlation between cell killing and residual chromatin breaks measured by PCC in six human cell lines irradiated with different radiation types. Int J Radiat Biol 2000;76:1189-96.

42. Elsasser $T$, Kramer M, Scholz M. Accuracy of the local effect model for the prediction of biologic effects of carbon ion beams in vitro and in vivo. Int J Radiat Oncol Biol Phys 2008;71:866-72.

43. Uzawa A, Ando K, Koike $S$, et al. Comparison of biological effectiveness of carbon-ion beams in Japan and Germany. Int J Radiat Oncol Biol Phys 2009;73:1545-51.

44. Gueulette J, Gregoire V, Octave-Prignot M, Wambersie A. Measurements of radiobiological effectiveness in the $85 \mathrm{MeV}$ proton beam produced at the cyclotron CYCLONE of Louvainla-Neuve, Belgium. Radiat Res 1996;145:70-4.

45. Gerweck LE, Kozin SV. Relative biological effectiveness of proton beams in clinical therapy. Radiother Oncol 1999;50: 135-42.

46. Blattmann H. Beam delivery systems for charged particles. Radiat Environ Biophys 1992;31:219-31.

47. Scholz M, Kellerer AM, Kraft-Weyrather W, Kraft G. Computation of cell survival in heavy ion beams for therapy: the model 
and its approximation. Radiat Environ Biophys 1997;36:59-66. 48. Baek HJ, Kim TH, Shin D, et al. Radiobiological characterization of proton beam at the National Cancer Center in Korea. J Radiat Res 2008;49:509-15.
49. Kim SS, Choo DW, Shin D, et al. In vivo radiobiological characterization of proton beam at the National Cancer Center in Korea: effect of the Chk2 mutation. Int J Radiat Oncol Biol Phys 2011;79:559-62. 